\title{
Clinical Aspects of Periungual Desquamation in Kawasaki Disease
}

\author{
Sung Hoon Kim, ${ }^{1}$ Hae Jeong Lee, ${ }^{1}$ and Ju Suk Lee ${ }^{1, *}$ \\ ${ }^{1}$ Department of Pediatrics, Samsung Changwon Hospital, Sungkyunkwan University School of Medicine, Changwon, Korea \\ "Corresponding author: Ju Suk Lee, MD, PhD, Department of Pediatrics, Samsung Changwon Hospital, Sungkyunkwan University School of Medicine, 158 Palyong - ro, Masan \\ Hoiwon - gu, Changwon - City, Gyeongsangnam - do, 51353, South Korea. Tel: +82-552335310, E-mail: ljs8952194@nate.com \\ Received 2017 July 31; Revised 2018 January 20; Accepted 2018 March 13.
}

\begin{abstract}
Objectives: To assess the relationship between coronary artery changes and periungual desquamation in Kawasaki disease (KD) and non - KD patients.

Methods: This retrospective descriptive study was conducted through a review of records of children with KD from March 2011 to February 2016. We also analyzed non - KD subjects with desquamation.

Results: Among 329 patients with KD, 177 (53.8\%) had periungual desquamation. Subjects with desquamation had longer duration of fever ( $6.75 \pm 2.43$ vs. $5.63 \pm 1.78$ days), more frequent erythema and edema of hands/feet (72.9\% vs. 51.9\%), and more frequent rash (94.4\% vs. $74.3 \%)$ than subjects without desquamation. They also had a higher platelet count (470420 \pm 127163 vs. $351240 \pm 105836, \mathrm{P}$ $=0.042$ ) and higher levels of aspartate aminotransferase (AST; $91.58 \pm 62.89 \mathrm{vs.} 55.5 \pm 44.72 \mathrm{IU} / \mathrm{L}, \mathrm{P}=0.031$ ), alanine aminotransferase (ALT; $105.39 \pm 71.54$ vs. $76.7 \pm 91.25 \mathrm{IU} / \mathrm{L}, \mathrm{P}=0.029$ ), and pro - brain natriuretic peptide (BNP; $1738.24 \pm 584.26$ vs. $1034.65 \pm 754.25$ $\mathrm{pg} / \mathrm{mL}, \mathrm{P}=0.042$ ). There was no difference between the two groups in development of coronary artery changes. We also analyzed 47 non - KD subjects with desquamation. These patients had fewer clinical signs (87\% had one sign of KD) and shorter fever duration according to parents' reported history, and had a lower platelet count, AST, ALT, and BNP than patients with KD. These patients had no coronary artery lesions.

Conclusions: Presence of periungual desquamation is not helpful for an early diagnosis of KD and is not an independent predictor of coronary artery changes in patients with or without KD.
\end{abstract}

Keywords: Kawasaki Disease, Periungual Desquamation, Coronary Artery

\section{Background}

Kawasaki disease (KD) is an acute febrile vasculitis that predominantly affects children under 5 years of age (1, $2)$. Dr. Kawasaki identified periungual desquamation in 49 of his 50 original patients; this phenomenon is seen in the subacute phase of $\mathrm{KD}$, typically 2 to 3 weeks after the onset of fever $(1,2)$. Periungual desquamation is not helpful in making an early diagnosis of KD but clinicians often consider the diagnosis of missed or self - recovered KD in a child with an antecedent prolonged pyrexia and late periungual desquamation. In particular, infants younger than 6 months often have incomplete presentations of KD with transient or subtle signs and symptoms, and these infants have a high risk for developing coronary artery changes (2). Therefore, although other conditions such as scarlet fever, sunburn, and side effects of medications can cause periungual desquamation, clinicians and parents usually consider the possibility of coronary arterial changes associated with missed KD in patients with periungual desquamation even though the sensitivity and specificity of this clinical phenomenon are unknown. The purpose of this study was to summarize the clinical features of KD with periungual desquamation and to investigate the relationship between changes in coronary artery lesions and desquamation. We also compared KD with desquamation and non - KD with desquamation to investigate the possibility of desquamation as a predictive factor of coronary artery changes in any clinical situation.

\section{Methods}

We retrospectively reviewed the records of patients who were discharged from Samsung Changwon Hospital with a diagnosis of KD between March 2011 and February 2016. Patients who were referred from another hospital for only echocardiography after treatment of KD were excluded because laboratory results at the time of treatment were not available. Patients with no follow up after discharge regardless of cause were also excluded. According to the 2004 American Heart Association (AHA) guidelines 
for the diagnosis of $\mathrm{KD}$, a diagnosis of complete $\mathrm{KD}$ (cKD) was based on clinical criteria, which included fever lasting 5 days or longer and four or more of the following five features: (1) bilateral conjunctival injection; (2) oral mucosa changes; (3) cervical lymphadenopathy $(>1.5 \mathrm{~cm}$ in diameter); (4) polymorphous rashes; (5) swelling or redness of the extremities, in addition to the exclusion of alternative diagnoses (2). Patients who did not perfectly meet the clinical criteria were diagnosed as incomplete KD (iKD) if they had a persistent fever that lasted 5 days or longer but with fewer than four of the other features and if other possible causes of fever had been excluded (2). According to the guideline, echocardiogram was considered positive if any of the following conditions were met: (1) z score of left anterior descending coronary artery (LAD) or right coronary artery $(\mathrm{RCA}) \geq 2.5$, (2) coronary arteries meeting the Japanese Ministry of Health criteria for aneurysms if the internal lumen diameter was $>3 \mathrm{~mm}$ in children $<5$ years old or $>4 \mathrm{~mm}$ in children $\geq 5$ years old, or (3) presence of three or more other suggestive features, including lack of tapering, decreased LV function, mitral regurgitation, pericardial effusion, or z scores in LAD or RCA of 2 2.5 (2). All patients were examined in the KD clinic after discharge by a single pediatric cardiologist (Dr. SH Kim). Our KD clinic policy for follow - up visits was as follows: the first examination in the outpatient clinic was 1 week after discharge, the second was 8 weeks and the third was 3 months after fever onset. In the outpatient clinic, fingers and toes were examined and the presence or absence of periungual desquamation and nail bed changes were described by a single pediatric cardiologist at all three visits. The parents were interviewed regarding the presence and extent of periungual desquamation. The severity of periungual desquamation was discretionally classified as mild (desquamation extended around the digit tip) or severe (extended to palm) according to examination by a cardiologist or interview with parents. Patients' clinical characteristics and past history, laboratory results, response to intravenous immunoglobulin (IVIG), length of hospital stay, and coronary artery outcome were reviewed and compared between two groups using independent t - test, Pearson's Chi - Square test, and Fisher's exact test. All statistical analyses were performed with IBM SPSS ver. 21.0 software (IBM Inc., Chicago, IL, USA). All values with $\mathrm{P}<0.05$ were considered statistically significant and all parameters were expressed as mean \pm standard deviation. During the same period, we analyzed 65 patients with an antecedent prolonged fever with late periungual desquamation but no diagnosis of KD. Among them, 18 patients were excluded because there was no laboratory data, therefore a total of 47 patients were enrolled in this study. As these patients had outpatient status, the medical records for fever duration and clinical signs depended on history provided by the parents and there were no data for initial laboratory tests when patients were in a febrile state. Instead, they underwent laboratory testing and echocardiography when they first visited the outpatient clinic. Their laboratory findings were compared with those of patients with KD with desquamation at the 1 - week follow up after discharge. Approval of this retrospective study was obtained from the institutional review board of our institution.

\section{Results}

From March 2011 to February 2016, a total of 347 children were diagnosed with KD, of which 118 (34\%) patients had iKD. After exclusion of 18 patients because of follow up loss, a total of 329 patients during this 5 - year period met enrollment criteria. Of these, 108 (32.8\%) were diagnosed as iKD and 177 (53.8\%) had periungual desquamation of either the fingers or toes. Desquamation of the fingers only was noted in 116 (65.5\%) patients, toes only in 13 (7.3\%) patients, and both fingers and toes in 48 (27.1\%). Discretionally classified severity of desquamation was mild type in 132 (74.6\%) patients and severe type in 45 (25.4\%) patients. Onset time of desquamation was $19.14 \pm 3.5$ days (range 8 to 27 days) from first onset of fever and $11.27 \pm$ 4.6 days (range 2 to 16 days) from last onset of fever after treatment. Subjects with and without desquamation were similar with respect to gender, age, onset of bilateral conjunctival injection, oropharyngeal changes (such as red lips and strawberry tongue), presence of cervical lymphadenopathy, and BCG site redness. Subjects with desquamation had longer duration of fever $(6.75 \pm 2.43$ vs. $5.63 \pm 1.78$ days, $\mathrm{P}=0.021)$, more frequent erythema and edema of hands/feet (72.9\% vs. 51.9\%, $\mathrm{P}=0.032)$, and more frequent rash $(94.4 \%$ vs. $74.3 \%, \mathrm{P}=0.013)$ than subjects without desquamation (Table 1). In the laboratory findings, subjects with and without desquamation were similar with respect to hemoglobin $(\mathrm{Hb})$, white blood cell (WBC) counts, percentage of bands, serum electrolytes, albumin, erythrocyte sedimentation rate (ESR), and C reactive protein (CRP). Subjects with desquamation had a higher platelet count $(470420 \pm 127163$ vs. $351240 \pm$ 105836, $\mathrm{P}=0.042$ ), higher plasma concentrations of aspartate aminotransferase (AST; $91.58 \pm 62.89$ vs. $55.5 \pm 44.72$ $\mathrm{IU} / \mathrm{L}, \mathrm{P}=0.031$ ) and alanine aminotransferase (ALT;105.39 \pm 71.54 vs. $76.7 \pm 91.25 \mathrm{IU} / \mathrm{L}, \mathrm{P}=0.029$ ), and higher pro-brain natriuretic peptide (proBNP;1738.24 \pm 584.26 vs. $1034.65 \pm$ $754.25 \mathrm{pg} / \mathrm{mL}, \mathrm{P}=0.042$ ) on admission (Table 2 ). There was no difference between the two groups regarding response to IVIG treatment and development of coronary artery lesions such as coronary aneurysm. In addition, the severity 
of desquamation was not related to any difference in clinical signs, laboratory findings, response to IVIG treatment, and coronary artery lesions.

\begin{tabular}{|c|c|c|}
\hline & $\begin{array}{l}\text { Desquamation } \\
\quad(\mathbf{N}=177)\end{array}$ & $\begin{array}{c}\text { No Desqua- } \\
\text { mation }(\mathrm{N}= \\
\mathbf{1 5 2})\end{array}$ \\
\hline CKD: iKD & 132: 45 & 89: 63 \\
\hline Male/Female & $107 / 70$ & $62 / 90$ \\
\hline Age (months) & $30.86 \pm 25.02$ & $37.31 \pm 45.6$ \\
\hline Duration of fever (days) & $6.75 \pm 2.43$ & $5.63 \pm 1.78$ \\
\hline Conjunctival injection, no. (\%) & $153(88.7)$ & $139(91.4)$ \\
\hline Oropharyngeal changes, no. (\%) & $152(85.9)$ & $123(80.9)$ \\
\hline $\begin{array}{l}\text { Hand \& foot erythema and edema, no. } \\
\text { (\%) }\end{array}$ & $129(72.9)$ & $79(51.9)$ \\
\hline Rash, no. (\%) & $167(94.4)$ & $113(74.3)$ \\
\hline BCG site redness, no. (\%) & $49(27.7)$ & $35(23)$ \\
\hline Cervical lymphadenopathy, no. (\%) & $38(21.5)$ & $33(21.7)$ \\
\hline \multicolumn{3}{|l|}{ Response to IVIG } \\
\hline $2^{\text {nd }}$ use of IVIG, no. (\%) & $22(12.4)$ & $21(13.8)$ \\
\hline Steroid use, no. (\%) & $8(4.5)$ & $3(1.9)$ \\
\hline Infliximab use, no. (\%) & $1(0.5)$ & $1(0.6)$ \\
\hline
\end{tabular}

Abbreviations: cKD, complete Kawasaki disease; iKD, incomplete Kawasaki disease; IVIG, intravenous immunoglobulin.

For the 47 non - KD subjects with an antecedent prolonged fever with later periungual desquamation, in all cases the reason for visiting our KD clinic was concern about the possibility of missed KD; $47.2 \%$ of these patients were referred by primary pediatricians, $23.6 \%$ were referred by a dermatologist, and the remaining $29.2 \%$ visited as a result of the parent's decision based on internet searches. The mean age was $47.19 \pm 52.37$ months (range 3 to 119 months). These patients had fewer clinical signs ( $87 \%$ of patients had one sign and $11 \%$ had two signs of KD, and the most common sign with previous fever was erythematous macular rash in 61.4\%), a shorter fever duration according to parent's history, and a lower platelet count and lower serum levels of AST, ALT, and proBNP than patients with KD with desquamation (Table 3). It is wellknown that several conditions can mimic KD (2), including infections (Epstein - Barr virus, adenovirus, measles), toxin - mediated illnesses (toxic shock syndrome, scarlet fever), inflammatory conditions (systemic juvenile idiopathic arthritis), and drug reactions (Stevens Johnson syndrome) but it was not easy to find the cause of antecedent fever in the chart review. Onset time of desquamation was $18.2 \pm 4.12$ days (range 7 to 35 days) from first onset of fever and $13.72 \pm 4.6$
Table 2. Laboratory Characteristics of Kawasaki Disease Patients with or without Periungual Desquamation

\begin{tabular}{lcc}
\hline & $\begin{array}{c}\text { Desquamation } \\
(\mathbf{N}=\mathbf{1 7 7})\end{array}$ & $\begin{array}{c}\text { No } \\
\text { Desquamation } \\
(\mathbf{N}=\mathbf{1 5 2})\end{array}$ \\
\hline Hb & $11.27 \pm 1.12$ & $11.49 \pm 0.96$ \\
\hline WBC $(/ \mu \mathbf{L})$ & $13892 \pm 4398$ & $14067 \pm 4498$ \\
\hline Band forms $(\%)$ & $58.01 \pm 18.16$ & $63.88 \pm 12.81$ \\
\hline Platelets $(/ \mu \mathbf{L})$ & $470420 \pm 127163$ & $351240 \pm 105836$ \\
\hline ESR $(\mathbf{m m} / \mathbf{h r})$ & $44.35 \pm 32.75$ & $41.28 \pm 35.67$ \\
\hline CRP $(\mathbf{m g} / \mathbf{L})$ & $67.84 \pm 63.01$ & $72.29 \pm 52.18$ \\
\hline AST $(\mathbf{I U} / \mathbf{L})$ & $91.58 \pm 62.89$ & $55.5 \pm 44.72$ \\
\hline ALT $(\mathbf{I U} / \mathbf{L})$ & $105.39 \pm 71.54$ & $76.7 \pm 91.25$ \\
\hline ProBNP $(\mathbf{p g} / \mathbf{m L})$ & $1738.24 \pm 584.26$ & $1034.65 \pm 754.25$ \\
\hline Coronary artery change & & $143(94.1)$ \\
\hline \multicolumn{1}{c}{ Normal } & $165(93.2)$ & $8(5.2)$ \\
\hline Transient ectasia & $9(5.1)$ & $1(0.66)$ \\
\hline Aneurysm & $3(1.7)$ & \\
\hline
\end{tabular}

Abbreviations: ALT, alanine aminotransferase; AST, aspartate aminotransferase; CRP, c - reactive protein; ESR, erythrocyte sedimentation rate; ProBNP, pro - brain natriuretic peptide; WBC, white blood cell.

days (range 4 to 27 days) from last onset of fever according to interview with the parents. Almost all of the patients had the mild type of severity $(\mathrm{N}=45,95.7 \%)$ and desquamation was predominantly present in the fingers $(\mathrm{N}=39$, $82.9 \%)$, and to a lesser extent in the toes $(\mathrm{N}=7,14.9 \%)$ and fingers and toes $(\mathrm{N}=1,2.1 \%)$. No coronary artery lesions were detected on echocardiography.

Table 3. Laboratory Characteristics of Kawasaki Disease Patients with Periungual Desquamation and Non KD with Periungual Desquamation ${ }^{\mathrm{a}}$

\begin{tabular}{lcc}
\hline & $\begin{array}{c}\text { KD with } \\
\text { Desquamation }\end{array}$ & $\begin{array}{c}\text { Non KD with } \\
\text { Desquamation }\end{array}$ \\
\hline Hb & $11.27 \pm 1.12$ & $11.93 \pm 2.19$ \\
\hline WBC & $7672 \pm 2418$ & $6483 \pm 1935$ \\
\hline Band forms & $37.17 \pm 21.35$ & $36.29 \pm 12.87$ \\
\hline Platelets & $581617 \pm 157219$ & $234712 \pm 131690$ \\
\hline ESR & $14.47 \pm 22.6$ & $13.97 \pm 19.24$ \\
\hline CRP & $17.51 \pm 10.17$ & $11.98 \pm 11.13$ \\
\hline AST & $56.54 \pm 22.58$ & $21.25 \pm 15.75$ \\
\hline ALT & $45.19 \pm 31.62$ & $20.98 \pm 21.45$ \\
\hline proBNP & $1038.71 \pm 616.36$ & $101.45 \pm 78.26$ \\
\hline
\end{tabular}

Abbreviations: ALT, alanine aminotransferase; AST, aspartate aminotransferase; CRP, c - reactive protein; ESR, erythrocyte sedimentation rate; ProBNP, pro - brain natriuretic peptide; WBC, white blood cell.

${ }^{\mathrm{a}}$ Non - KD: patients with antecedent fever but not diagnosed as a Kawasaki disease.

${ }^{\mathrm{b}}$ Laboratory findings checked one week after discharge. 


\section{Discussion}

$\mathrm{KD}$ is an acute febrile systemic vasculitis of unknown etiology, mainly affecting children younger than 5 years (1). Despite a decrease in the number of children in South Korea due to the low birth rate, the incidence of KD in children $<5$ years of age has shown a marked increase and is estimated to have increased to 134.4 per 100000 children $<5$ years of age in South Korea in 2011 (3). This incidence is the second highest rate in the world after Japan, where the incidence of $\mathrm{KD}$ in children $<5$ years of age was 239.6 per 100000 children in 2010 (4). It is likely that the number of cases of KD actually has increased, however several factors might influence these data such as increased awareness of KD by physicians and especially parents, who can now easily find information about KD by internet searches; the background of the shared racial characteristics of South Koreans and Japanese and the similar climates of South Korea and Japan; and earlier application of echocardiographic examinations for the detection of coronary artery changes (3-6). Although coronary aneurysm occurred in only 1.9\% of KD in Korea (3), it is easy to understand that physicians and parents worry about coronary artery changes of missed KD in children with antecedent fever with later periungual desquamation. In our results, we experienced 65 patients with antecedent fever and later periungual desquamation who all worried about missed or self - recovered KD during the same surveillance period. Many studies have reported that periungual desquamation in KD is seen in the subacute phase, typically 2 to 3 weeks after the onset of fever $(1,2,5,7,8)$. Several earlier published reports mentioned the incidence of desquamation $(1,9,10)$ but there are few recent reports of its incidence $(11,12)$. In our study, $177(53.8 \%)$ of KD patients had periungual desquamation of either the fingers or toes. This rate is lower than previously reported values of $98 \%, 93 \%$, and $68 \%$, respectively $(1,10,12)$ but slightly higher than the most recently report $(50.5 \%<1$ year, $40.5 \%$ $>1$ year)(11). This difference in the incidence rate may be influenced by many factors. First, in the study period of previously cited reports, KD was a new disease and there was no guideline for diagnosis and treatment, and the authors were especially interested in describing the clinical manifestations and coronary artery changes of $\operatorname{KD}(1,10)$. Until uniform treatment was established, patients were not effectively treated by anti - inflammatory therapy during this period. Periungual desquamation is seen in the typically 2 to 3 weeks after the onset of fever, therefore this phenomenon is now observed in outpatient services and the detection rate is dependent on the parent's observation and memory. According to recent studies, the incidence of incomplete $\mathrm{KD}$ is increasing $(3,5,6,11)$ and this may also influence the onset of desquamation. Finally, the detection rate may be influenced by the observation method, e.g. with a lighted magnifying lens (12). Although there was a single report that subjects who did not show peeling were more likely to develop coronary artery aneurysm (12), our study revealed that the presence or absence of peeling was not related to coronary artery changes in KD. Although there are two reports of a child with atypical course and a few clinical signs of KD who had later periungual desquamation with development of coronary artery aneurysm (13, 14 ), on the basis of our results we may draw the following conclusion: a child with peeling who has relatively short duration of antecedent fever with few clinical signs of KD (in our study, $87 \%$ of subjects had only one sign), no thrombocytosis, and normal range of AST, ALT and proBNP is less likely to have coronary artery changes.

Limitations of our study include the retrospective nature of the medical records review and the different timing of visits after the onset of fever in KD and non - KD subjects. Moreover, it is the experience of only one center and does not reflect nationwide incidence rates or relationships between coronary artery changes and desquamation in KD. In particular, our study had a lower incidence of coronary dilatation and aneurysm than reported in a nationwide survey during 2009 - 2011 in South Korea (5.16\% vs. $16.4 \%, 1.2 \%$ vs. $1.9 \%$, respectively) (3). In some cases, determination of the presence or absence of periungual desquamation was dependent on only the parent's reported history or on the physician's direct examination, which might have variable accuracy. It was difficult to find the cause of antecedent fever in the non - KD cases with desquamation from the chart review. Despite these limitations, our data reveal that there is no relationship between periungual desquamation and coronary artery changes regardless of KD.

\subsection{Conclusion}

The presence of periungual desquamation is not an independent predictor of coronary artery changes in KD or non - KD patients. Clinicians should be cautioned not to alarm parents of a non - KD child with peeling with respect to the possibility of coronary artery change.

\section{References}

1. Kawasaki T, Kosaki F, Okawa S, Shigematsu I, Yanagawa H. A new infantile acute febrile mucocutaneous lymph node syndrome (MLNS) prevailing in Japan. Pediatrics. 1974;54(3):271-6. [PubMed: 4153258].

2. Newburger JW, Takahashi M, Gerber MA, Gewitz MH, Tani LY, Burns JC, et al. Diagnosis, treatment, and long-term management of Kawasaki disease: a statement for health professionals from the Committee on Rheumatic Fever, Endocarditis, and Kawasaki Disease, Council on Cardiovascular Disease in the Young, American Heart Association. 
Pediatrics. 2004;114(6):1708-33. doi: 10.1542/peds.2004-2182. [PubMed: 15574639].

3. Kim GB, Han JW, Park YW, Song MS, Hong YM, Cha SH, et al. Epidemiologic features of Kawasaki disease in South Korea: data from nationwide survey, 2009-2011. Pediatr Infect Dis J. 2014;33(1):24-7. doi: 10.1097/INF.0000000000000010. [PubMed: 24064559].

4. Nakamura Y, Yashiro M, Uehara R, Sadakane A, Tsuboi S, Aoyama Y, et al. Epidemiologic features of Kawasaki disease in Japan: results of the 2009-2010 nationwide survey. J Epidemiol. 2012;22(3):216-21. [PubMed: 22447211]. [PubMed Central: PMC3798622].

5. Lue HC, Chen LR, Lin MT, Chang LY, Wang JK, Lee CY, et al. Epidemiological features of Kawasaki disease in Taiwan, 1976-2007: results of five nationwide questionnaire hospital surveys. Pediatr Neonatol.2014;55(2):92-6. doi:10.1016/j.pedneo.2013.07.010. [PubMed: 24120536].

6. Saundankar J, Yim D, Itotoh B, Payne R, Maslin K, Jape G, et al. The epidemiology and clinical features of Kawasaki disease in Australia. Pediatrics.2014;133(4):e1009-14. doi:10.1542/peds.2013-2936. [PubMed: 24685950].

7. Bayers S, Shulman ST, Paller AS. Kawasaki disease: part I. Diagnosis, clinical features, and pathogenesis.JAm Acad Dermatol.2013;69(4):501 e1-11. quiz 511-2. doi: 10.1016/j.jaad.2013.07.002. [PubMed: 24034379]

8. Yim D, Curtis N, Cheung M, Burgner D. An update on Kawasaki dis- ease II: clinical features, diagnosis, treatment and outcomes. J Paediatr Child Health. 2013;49(8):614-23. doi: 10.1111/jpc.12221. [PubMed: 23647873].

9. Ichida F, Fatica NS, O'Loughlin JE, Klein AA, Snyder MS, Levin AR, et al. Epidemiologic aspects of Kawasaki disease in a Manhattan hospital. Pediatrics. 1989;84(2):235-41. [PubMed: 2748250].

10. Morens DM, Anderson LJ, Hurwitz ES. National surveillance of Kawasaki disease. Pediatrics. 1980;65(1):21-5. [PubMed: 7355030].

11. Teng MC, Wang LC, Yu HH, Lee JH, Yang YH, Chiang BL. Kawasaki disease and Henoch-Schonlein purpura - 10 years' experience of childhood vasculitis at a university hospital in Taiwan. J Microbiol Immunol Infect. 2012;45(1):22-30. doi: 10.1016/j.jmii.2011.09.024. [PubMed: 22177369].

12. Wang S, Best BM, Burns JC. Periungual desquamation in patients with Kawasaki disease. Pediatr Infect Dis J. 2009;28(6):538-9. doi: 10.1097/INF.ob013e3181945984. [PubMed: 19483521]. [PubMed Central: PMC2738931].

13. Kuijpers TW, Tjia KL, de Jager F, Peters M, Lam J. A boy with chickenpox whose fingers peeled. Lancet. 1998;351(9118):1782. doi: 10.1016/S01406736(98)04021-5. [PubMed: 9635952].

14. Mitra S, Singh S, Grover A, Kumar L. A child with prolonged pyrexia and peripheral desquamation: is it Kawasaki disease? Indian Pediatr. 2000;37(7):786-9. [PubMed: 10906815]. 